\title{
SIMULAÇÃO DE DESDOBRAMENTOS COMPLEXOS DE ESPECTROS DE RMN DE ${ }^{1} H$
}

\author{
Mauricio Gomes Constantino*, Gil Valdo José da Silva, Vladimir Constantino Gomes Heleno e Ivana Aparecida Borin \\ Departamento de Química, Faculdade de Filosofia, Ciências e Letras de Ribeirão Preto, Universidade de São Paulo, \\ Av. Bandeirantes, 3900, 14040-901 Ribeirão Preto - SP \\ Ivan P. de Arruda Campos \\ Instituto de Ciências Exatas e Tecnologia, Universidade Paulista, Rua Dr. Bacelar, 1212, 04026-002 São Paulo - SP
}

Recebido em 10/2/05; aceito em 20/5/05; publicado na web em 01/12/05

\begin{abstract}
SIMULATION OF COMPLEX SPLITTINGS OF ${ }^{1} \mathrm{H}$ NMR SPECTRA. Complex ${ }^{1} \mathrm{H}$ NMR spectra multiplets that cannot be easily understood by simple inspection are rather frequent in the daily work of the organic chemistry analyst. The multiple and excellent new techniques available from modern instruments usually provide satisfactory solutions, but there are still many cases where a simulation is necessary, at least to obtain a final confirmation. It is extremely convincing to see that a graph, obtained by calculations with chemical-shift and coupling-constant values only, can be virtually identical to the experimental spectrum. This paper describes a computer program to make such calculations. The program is free and can be downloaded from http://artemis.ffclrp.usp.br/ NMR.htm (click on SimEsp_NMR_Compil.zip). All routines are also available and may be used without any restrictions. The paper includes a fairly detailed discussion about how the calculations are made.
\end{abstract}

Keywords: computer program; ${ }^{1} \mathrm{H}$ NMR; spectrum simulation.

\section{INTRODUÇÃO}

Os espectros de ressonância magnética nuclear, tanto de ${ }^{1} \mathrm{H}$ como de ${ }^{13} \mathrm{C}$, são hoje as ferramentas mais poderosas para elucidação da estrutura molecular das substâncias orgânicas. A análise de um espectro (especialmente de $\mathrm{RMN}$ de ${ }^{1} \mathrm{H}$ ), porém, freqüentemente requer um minucioso estudo dos desdobramentos das bandas para que o máximo de informações possa ser obtido dos dados experimentais. Mesmo com a utilização das inúmeras e eficientes técnicas que os modernos aparelhos permitem, ainda há circunstâncias em que a simulação de um desdobramento complexo é muito importante, no mínimo para trazer uma confirmação final; é extremamente convincente observar que um gráfico, produzido apenas por cálculos com os valores de deslocamentos químicos e de constantes de acoplamento, pode ser virtualmente idêntico ao espectro obtido experimentalmente. $\mathrm{O}$ alto poder de resolução dos aparelhos atualmente disponíveis leva a grande maioria dos sinais a apresentarem características relativamente simples de espectros de primeira ordem; para estes casos, uma simulação com programas como o FOMSC $3^{1}$ é suficiente. Em nossa pesquisa temos encontrado, porém, ocasionalmente, casos de desdobramentos de ordem superior (veja exemplo adiante), o que nos animou a desenvolver o programa SimEsp_NMR que ora apresentamos e descrevemos de forma sumária.

Desde pouco depois da descoberta do fenômeno da RMN, inúmeros programas de computador foram desenvolvidos para simulação de espectros. Devemos, porém, notar que há grande diferença de objetivos entre esses programas: alguns se destinam a fazer previsões de deslocamentos químicos baseados na estrutura molecular (suposta ou comprovada), outros se destinam a prever o aspecto de um espectro quando são conhecidos os deslocamentos químicos e os valores das constantes de acoplamento. O programa SimEsp_NMR pertence a este último tipo; pode ser facilmente utilizado para fazer confirmações, mas pode também, com alguma

*e-mail: mgconsta@usp.br habilidade e paciência, ser usado para determinar ou refinar valores de deslocamentos químicos e constantes de acoplamento. $\mathrm{Na}$ forma em que está (mas que pode ser modificada pelo usuário), o programa pode trabalhar com até 10 núcleos (hidrogênios).

Sua finalidade principal, porém, é de caráter didático e demonstrativo. As três características principais deste programa são: foi desenvolvido para os computadores mais populares hoje em nosso meio universitário, com plataformas Windows; o programa é aberto: suas rotinas estão à disposição de qualquer interessado, na forma do programa completo em Visual Basic ou, para quem utiliza outras linguagens e não disponha do Visual Basic, as principais rotinas estão também disponíveis na forma de arquivos de texto (em Basic; o interessado terá que traduzí-las) e, o programa contém informações de caráter demonstrativo: é possível visualizar as matrizes, observar detalhes das operações de cálculo de intensidades, e são mostrados vários cálculos que podem ser muito úteis para verificar se as rotinas estão trabalhando corretamente.

Além dessas há várias outras características particulares: o gráfico $^{2}$ do resultado pode conter (ou não) um histograma colorido, que identifica as bandas provenientes de cada hidrogênio pela cor, além de uma envolvente lorentziana e/ou gaussiana (na envolvente aparecem assinalados, no gráfico, os máximos locais relativos, com os correspondentes deslocamentos químicos em $\mathrm{Hz}$ ); o gráfico pode ser copiado e colado em programas editores de texto, como o Word, para impressão de alta qualidade (é um arquivo "metafile", não o tipo comum de "bitmap", que não tem boa resolução para impressão). O resultado é também apresentado na tela na forma de tabela, que também pode ser copiada e colada em outros programas, como o Excel ou o Word; clicando com o mouse em uma linha da tabela, uma seta aparece no gráfico, indicando a linha correspondente do histograma (e vice-versa, clicando em uma linha do gráfico, na tabela é mostrada a linha correspondente).

O uso do programa e de suas rotinas é inteiramente livre: o leitor interessado pode abrir a página http://artemis.ffclrp.usp.br/ NMR.htm e baixar quaisquer dos seguintes arquivos ${ }^{3}$ :

1. SimEsp_NMR_Compil.zip (tamanho 2,96 MB): programa com- 
pilado, pronto para ser instalado e usado em qualquer computador com plataforma Windows.

2. SimEsp_NMR_Basic.zip (tamanho $201 \mathrm{~KB}$ ): programa completo; só pode ser aberto se o leitor dispuser do programa Visual Basic. Neste caso, todas as rotinas estarão disponíveis para serem utilizadas em outros programas, para serem adaptadas, aperfeiçoadas ou modificadas, etc.

3. SimEsp_NMR_texto(pdf).zip (tamanho $361 \mathrm{~KB}$ ): contém as principais rotinas em forma de arquivo de texto, para serem traduzidas e usadas ao gosto do leitor.

\section{DISCUSSÃO: COMO SÃO FEITOS OS CÁLCULOS}

\section{Introdução}

O programa foi desenvolvido para calcular espectros de RMN de ${ }^{1} \mathrm{H}$ (hidrogênio) e pode ser usado apenas para núcleos com número quântico de spin $I=1 / 2$. Tais núcleos podem existir em apenas dois estados de spin, caracterizados pelos números quânticos magnéticos $m=+1 / 2$ e $m=-1 / 2$; as funções de onda correspondentes são identificadas respectivamente pelas letras gregas $\alpha$ e $\beta^{4}$. Estes dois estados têm a mesma energia, isto é, são degenerados. Na presença de um campo magnético estático $B_{0}$, porém, os dois estados passam a ter energias diferentes como resultado da interação entre o momento magnético nuclear $(\mu)$ com o campo estático $B$. Um núcleo que esteja no nível mais baixo de energia (correspondendo a $\alpha$ ) pode, então, absorver energia radiante e passar para o nível mais alto de energia $(\beta)$, desde que a energia de um fóton desta radiação $(h v)$ seja igual à diferença de energia $\Delta E$ entre os dois estados $^{5}$.

Devido a certas simplificações que podem ser feitas no desenvolvimento das equações matemáticas, podemos suprimir a constante de Planck, $h$, de nossos cálculos e trabalhar apenas com a frequiência da radiação, $v$, interpretando que $v$ representa a diferença de energia entre os dois estados possíveis para um núcleo em um campo magnético. Uma coleção de moléculas contendo um único "tipo" de núcleo, se colocada em um campo magnético, pode absorver energia radiante de frequiência $v_{\mathrm{A}}$. $\mathrm{O}$ valor de $v_{\mathrm{A}}$ depende de $B_{\mathrm{o}} \mathrm{e}$, também, da densidade eletrônica em volta do núcleo, pois os elétrons em volta do núcleo geram um pequeno campo magnético contrário ao campo $B_{\mathrm{o}}$, neutralizando-o parcialmente. A freqüência de absorção de um hidrogênio é, por isso, uma função de sua vizinhança estrutural na molécula.

Moléculas que contenham $p$ tipos de hidrogênios (que não tenham interações uns com os outros) absorvem em $p$ freqüências diferentes. Essas diferenças são pequenas em relação ao valor absoluto das frequiências, e costuma-se utilizar o deslocamento químico para caracterizar uma certa absorção: o deslocamento químico seria a diferença entre a frequiência de absorção em questão e a frequiência de absorção dos hidrogênios da referência, o tetrametilsilano (TMS). Neste texto vamos representar o deslocamento químico (em Hz) por $\sigma$, e a freqüência de absorção do TMS (na prática, a "freqüência do instrumento") por $v_{0}$. Para um hidrogênio $i$, a freqüência de absorção é dada por

$v_{i}=v_{0}+\sigma_{i}$

Na entrada e saída de dados, os valores de "freqüência" são na realidade os valores $\sigma_{i}$ de deslocamento químico. Para efetuar os cálculos, porém, o computador utiliza os valores de frequiência "reais", $v_{i}$ : ao ler os valores de $\sigma_{i}$ fornecidos, a primeira providência tomada pelo programa é somar a cada um o valor de $v_{0}$; depois de feitos todos os cálculos, é subtraído o valor $v_{\mathrm{o}}$ de cada frequiência resultante, de forma que o resultado final é apresentado como deslocamento químico, $\sigma$. Quando há interação entre dois tipos de hidrogênios de uma mesma molécula o espectro se complica porque, apesar de o número de níveis de energia continuar a ser o mesmo, a energia de cada nível sofre alteração, e a igualdade que havia entre certas transições no caso de ser $J=0$, desaparece quando $J \neq 0$. Dizemos que as bandas simples sofrem um desdobramento. A finalidade principal deste programa é calcular qual seria a aparência de um espectro, se conhecemos os deslocamentos químicos $(\sigma)$ dos hidrogênios e o valor da interação entre eles (a constante de acoplamento, $J$ ).

A maneira como o programa efetua os cálculos pode ser melhor compreendida se antes examinarmos um sistema simples, AX, que pode ser considerado um sistema de primeira ordem (isto significa que o valor de $J$ é bem menor do que as diferenças entre os vários níveis de energia, o que permite que cálculos feitos de uma maneira bem simplificada resultem em soluções razoavelmente corretas). Nestas condições cada núcleo de hidrogênio pode existir em dois níveis de energia, que podem ser expressos da seguinte forma ${ }^{6}$ :

$E_{A \alpha}=-\left(+1 / 2 \vee_{A} h\right) \quad E_{A \beta}=-\left(-1 / 2 v_{A} h\right) \quad E_{X \alpha}=-\left(+1 / 2 v_{X} h\right) \quad E_{X \beta}=-\left(-1 / 2 v_{X} h\right)$

É claro que, se cada hidrogênio pode existir em dois níveis de energia, uma coleção de $p$ hidrogênios poderá existir em $2^{p}$ níveis diferentes. Para dois hidrogênios há 4 níveis: se $J=0$, as energias desses níveis podem ser calculadas simplesmente somando parcelas apropriadas escolhidas entre as 4 expressões acima. Dividindo todos os valores de energias pela constante de Planck $(h)$, ficamos com as seguintes expressões:

$\frac{E_{1}}{h}=\frac{E_{A \alpha}+E_{X \alpha}}{h}=-\left(+\frac{1}{2} \mathrm{v}_{A}+\frac{1}{2} \mathrm{v}_{X}\right)$

$\frac{E_{2}}{h}=\frac{E_{A \alpha}+E_{X \beta}}{h}=-\left(+\frac{1}{2} \mathrm{v}_{A}-\frac{1}{2} \mathrm{v}_{X}\right)$

$\frac{E_{3}}{h}=\frac{E_{A \beta}+E_{X \alpha}}{h}=-\left(-\frac{1}{2} v_{A}+\frac{1}{2} v_{X}\right)$

$\frac{E_{4}}{h}=\frac{E_{A \beta}+E_{X \beta}}{h}=-\left(-\frac{1}{2} v_{A}-\frac{1}{2} v_{X}\right)$

Quando $J \neq 0$, é necessário somar a cada nível o valor da energia do acoplamento spin-spin $E_{S S}$, que é dada pela seguinte expressão:

$\frac{E_{S S}}{h}=m_{A} m_{X} J_{A X}$

É evidente que, para $I=1 / 2$, o valor absoluto de $E_{S S}$ será sempre $1 / 4 J_{A X}$, sendo o seu sinal determinado pela combinação dos sinais de $m_{A}$, de $m_{X}$ e de $J$.

O diagrama da Figura 1 é bem auto-explicativo, e não há dificuldade em compreender como este é o resultado das equações apresentadas. É bem simples também verificar que, quando $J=0$, o espectro é mais simples mas não por envolver menor número de níveis ou de transições, mas simplesmente porque ocorrem igualdades que resultam em superposições de linhas. Essas igualdades desaparecem quando $J \neq 0$, e o espectro fica mais complexo. Naturalmente, para bem compreender o esquema da Figura 1, é necessário ter em mente que não são permitidas transições entre dois níveis quaisquer: a regra de seleção que se aplica é que somente são permitidas as transições entre dois níveis se $\Delta m_{T}= \pm 1$, sendo 


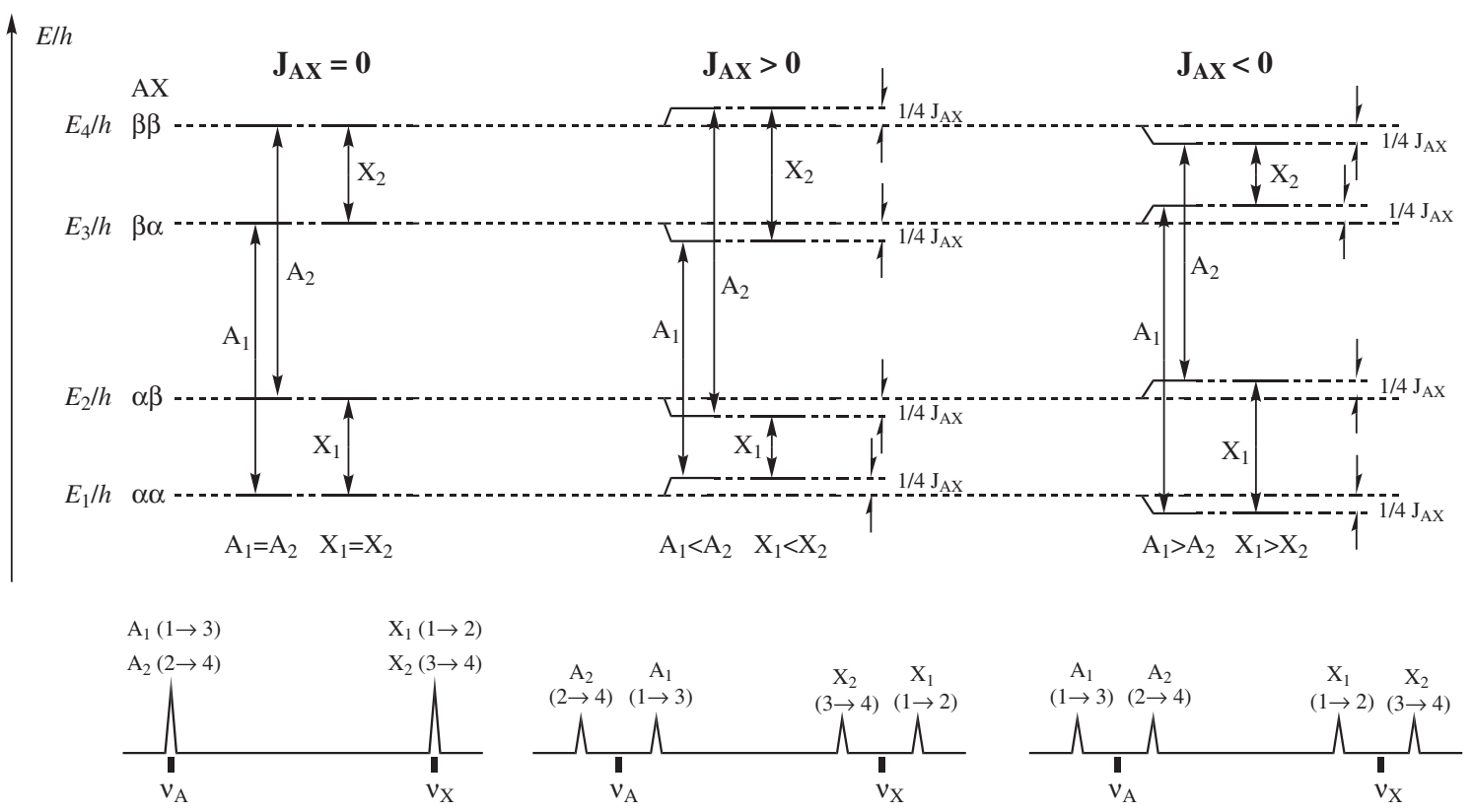

Figura 1. Diagrama de energias para sistema $A X$

$m_{T}$ a soma de todos os valores de $m$ para um certo nível. Assim não são permitidas transições entre o nível 1 e o nível $4\left(\Delta m_{T}=2\right)$ ou entre o nível 2 e o nível $3\left(\Delta m_{T}=0\right)$.

Generalizando, para um sistema com $p$ tipos diferentes de hidrogênios, a energia de cada nível pode ser calculada pela seguinte expressão (onde já abandonamos a constante de Planck, que não afetará os resultados nem as conclusões pois, como visto acima, aparecerá como divisor de todos os valores de energia):

$E(\mathrm{~Hz})=-\sum_{i=1}^{p} m_{i} v_{i}+\sum_{i=1}^{p-1} \sum_{j=i+1}^{p} m_{i} m_{j} J_{i j}$

Para cada um dos $2^{p}$ níveis possíveis de energia do sistema, devemos definir (arbitrar) o estado de spin $(m)$ para cada hidrogênio e em seguida devemos aplicar a Equação 1, encontrando o valor da energia deste nível (em Hz). Calculados todos os níveis, temos que aplicar a regra de seleção $\left(\Delta m_{T}= \pm 1\right)$ e calcular a diferença de energia para todos os pares de níveis em que a transição seja permitida. Chegamos assim a um histograma contendo uma linha para cada transição possível, com todas as linhas de mesma intensidade; aplicando as leis de distribuição (gaussiana e/ou lorentziana) chegamos a um espectro de primeira ordem.

\section{O espectro de ordem superior}

Quando os valores de $J$ se aproximam, em grandeza, dos valores das diferenças de energia entre os vários níveis, o espectro de primeira ordem fica muito diferente da realidade observada experimentalmente. Torna-se necessário, então, fazer cálculos bem mais complexos, utilizando os princípios da mecânica quântica, para obter aproximações realmente úteis. Não vamos entrar em detalhes teóricos, mas apenas apresentar os cálculos que o programa tem que efetuar para chegar ao espectro simulado. O leitor interessado não terá dificuldade de encontrar na literatura a fundamentação teórica apropriada ${ }^{7}$. As informações práticas apresentadas a seguir, por outro lado, são relativamente raras e dispersas na literatura e são essenciais para quem quiser modificar ou aperfeiçoar o programa para seus fins específicos.
Para calcular os níveis de energia, começamos por utilizar a mesma Equação 1 apresentada anteriormente; mas ela deve agora ser encarada como um operador hamiltoniano, usado para calcular os elementos da diagonal de uma matriz hamiltoniana. Para um sistema de $p$ hidrogênios, a matriz hamiltoniana $(H)$ é uma matriz quadrada $2^{p} \times 2^{p}$ cujos elementos da diagonal são, como já mencionado, calculados pela Equação 1; no programa, este cálculo é feito pelas rotinas LoopNi, FinLoopNi e LoopJ. A rotina FinLoopNi calcula também os valores de $m_{T}\left(\sum m_{i}\right)$ para cada nível, valores que serão úteis para cálculo dos elementos de fora da diagonal e para aplicação da regra de seleção.

Como exemplo, vamos tomar o caso de 3 hidrogênios $(p=3)$ : devemos fornecer ao programa os valores de deslocamento químico $\sigma_{1}, \sigma_{2}$ e $\sigma_{3}$ (com os quais serão calculadas as freqüências $v_{1}, v_{2}$ e $v_{3}$ ) bem como os valores das constantes de acoplamento $J_{12}, J_{13}$ e $J_{23}$. Teremos uma matriz $8 \times 8$ cujos elementos da diagonal serão como apresentado na Tabela 1 .

Estes valores são colocados em ordem crescente pela rotina ODMEL $^{8}$, de forma que teremos $H_{11}<H_{22}<H_{33}<\ldots<H_{88}$. Em seguida, a mesma rotina ODMEL calculará os elementos da matriz $H$ que estão fora da diagonal. A matriz tem um número de linhas (e de colunas) igual ao número de níveis de energia; para compreender os cálculos com maior facilidade deve-se entender que a linha 1 (assim como a coluna 1) corresponde ao nível de energia 1, a linha 2 (assim como a coluna 2) corresponde ao nível de energia 2, e assim por diante.

Para calcular os elementos de fora da diagonal, a rotina ODMEL utiliza dois critérios 9 . O primeiro é simples: se os níveis representados pela linha $k$ e pela coluna $l$ têm valores de spin total $\left(m_{T}\right)$ diferentes, o valor de $H_{k l}$ é zero. O segundo critério é mais elaborado: a rotina compara, para cada núcleo, o valor de $m$ nos dois níveis; o valor de $H_{k l}$ somente será diferente de zero se os dois níveis diferirem exclusivamente pelo valor de $m$ para dois núcleos, por ex., para os núcleos $i$ e $j$, caso em que o valor de $H_{k l}$ será $1 / 2 J_{\mathrm{ij}}$.

Como exemplo, digamos que a rotina esteja determinando $\mathrm{o}$ valor de $H_{k l}$, tendo já verificado que $m_{T}(k)=m_{T}(l)$ (portanto, de acordo com o primeiro critério, o elemento $H_{k l}$ pode ser diferente de zero). A rotina agora compara o valor de $m_{1}(k) \operatorname{com} m_{1}(l)$, de- 
Tabela 1. Cálculo dos elementos da diagonal da matriz $\mathrm{H}$

\begin{tabular}{|c|c|c|}
\hline & $m_{T}$ & \\
\hline$A_{11}=-\left(+\frac{-}{2} v_{1}+\frac{-}{2} v_{2}+\frac{-}{2} v_{3}\right)+\frac{-}{4} J_{12}+\frac{-}{4} J_{13}+\frac{-}{4} J_{23}$ & $+3 / 2$ & $\alpha \alpha \alpha$ \\
\hline$H_{22}=-\left(+\frac{1}{2} v_{1}+\frac{1}{2} v_{2}-\frac{1}{2} v_{3}\right)+\frac{1}{4} J_{12}-\frac{1}{4} J_{13}-\frac{1}{4} J_{23}$ & $+1 / 2$ & $\alpha \alpha \beta$ \\
\hline$H_{33}=-\left(+\frac{1}{2} v_{1}-\frac{1}{2} v_{2}+\frac{1}{2} v_{3}\right)-\frac{1}{4} J_{12}+\frac{1}{4} J_{13}-\frac{1}{4} J_{23}$ & $+1 / 2$ & $\alpha \beta \alpha$ \\
\hline$H_{44}=-\left(-\frac{1}{2} v_{1}+\frac{1}{2} v_{2}+\frac{1}{2} v_{3}\right)-\frac{1}{4} J_{12}-\frac{1}{4} J_{13}+\frac{1}{4} J_{23}$ & $+1 / 2$ & $\beta \alpha \alpha$ \\
\hline$H_{55}=-\left(+\frac{1}{2} v_{1}-\frac{1}{2} v_{2}-\frac{1}{2} v_{3}\right)-\frac{1}{4} J_{12}-\frac{1}{4} J_{13}+\frac{1}{4} J_{23}$ & $-1 / 2$ & $\alpha \beta \beta$ \\
\hline$H_{66}=-\left(-\frac{1}{2} v_{1}+\frac{1}{2} v_{2}-\frac{1}{2} v_{3}\right)-\frac{1}{4} J_{12}+\frac{1}{4} J_{13}-\frac{1}{4} J_{23}$ & $-1 / 2$ & $\beta \alpha \beta$ \\
\hline$H_{77}=-\left(-\frac{1}{2} v_{1}-\frac{1}{2} v_{2}+\frac{1}{2} v_{3}\right)+\frac{1}{4} J_{12}-\frac{1}{4} J_{13}-\frac{1}{4} J_{23}$ & $-1 / 2$ & $\beta \beta \alpha$ \\
\hline$H_{88}=-\left(-\frac{1}{2} v_{1}-\frac{1}{2} v_{2}-\frac{1}{2} v_{3}\right)+\frac{1}{4} J_{12}+\frac{1}{4} J_{13}+\frac{1}{4} J_{23}$ & $-3 / 2$ & $\beta \beta \beta$ \\
\hline
\end{tabular}

pois $m_{2}(k)$ com $m_{2}(l)$, e assim por diante. Somente quando forem satisfeitas as condições $m_{i}(k) \neq m_{i}(l)$ e $m_{j}(k) \neq m_{j}(l)$ e todos os demais valores de $m$ forem iguais nos dois níveis, só assim $H_{k l} \neq 0$; o valor será:

$H_{k l}=H_{l k}=\frac{1}{2} J_{i j}$

A matriz é simétrica, e para qualquer valor de $k$ e de $l$, $H_{k l}=H_{l k}$. Examinando a Tabela 1 (supondo-se que os valores de $H_{n n}$ estejam já ordenados em ordem crescente) não é difícil ver que, pelo primeiro critério, $H_{11}$ será o único valor diferente de zero na linha 1 e na coluna 1 , pois não há nenhum outro nível com $m_{T}=$ $+3 / 2$; o mesmo ocorre para a linha 8 e a coluna 8 , com apenas $H_{88}$ diferente de zero. Com o segundo critério podemos ver que:

$$
\begin{array}{ll}
H_{23}=H_{32}=\frac{1}{2} J_{23} & H_{56}=H_{65}=\frac{1}{2} J_{12} \\
H_{24}=H_{42}=\frac{1}{2} J_{13} & H_{57}=H_{75}=\frac{1}{2} J_{13} \\
H_{34}=H_{43}=\frac{1}{2} J_{12} & H_{67}=H_{76}=\frac{1}{2} J_{23}
\end{array}
$$

Todos os demais elementos são nulos, de modo que a matriz hamiltoniana fica como mostrado na Figura 2.

Os próximos passos são operações puramente matemáticas. Temos que resolver a matriz hamiltoniana, ou seja, determinar seus autovalores e os correspondentes autovetores. Os autovalores são os verdadeiros níveis de energia do sistema, e os autovetores, através das operações descritas mais adiante, fornecem as intensidades das bandas (ou a probabilidade das transições).

Como a matriz hamiltoniana é simétrica podemos utilizar, para resolvê-la, o método de Jacobi, que é um dos métodos mais eficientes e robustos para solução numérica do problema dos autovalores ${ }^{10}$. Essencialmente, as operações principais são as seguintes: os elementos da matriz $H$ são transferidos para uma matriz $A$, que é fornecida à rotina $\mathrm{JACOBI}$; esta cria uma matriz $V$, em que

$$
\begin{array}{l|cccccccc} 
& \mathbf{1} & \mathbf{2} & \mathbf{3} & \mathbf{4} & \mathbf{5} & \mathbf{6} & \mathbf{7} & \mathbf{8} \\
\mathbf{1} & H_{11} & 0 & 0 & 0 & 0 & 0 & 0 & 0 \\
\mathbf{2} & 0 & H_{22} & 1 / 2 J_{23} & 1 / 2 J_{13} & 0 & 0 & 0 & 0 \\
\mathbf{3} & 0 & 1 / 2 J_{23} & H_{33} & 1 / 2 J_{12} & 0 & 0 & 0 & 0 \\
\mathbf{4} & 0 & 1 / 2 J_{13} & 1 / 2 J_{12} & H_{44} & 0 & 0 & 0 & 0 \\
\mathbf{5} & 0 & 0 & 0 & 0 & H_{55} & 1 / 2 J_{12} & 1 / 2 J_{13} & 0 \\
\mathbf{6} & 0 & 0 & 0 & 0 & 1 / 2 J_{12} & H_{66} & 1 / 2 J_{23} & 0 \\
\mathbf{7} & 0 & 0 & 0 & 0 & 1 / 2 J_{13} & 1 / 2 J_{23} & H_{77} & 0 \\
\mathbf{8} & 0 & 0 & 0 & 0 & 0 & 0 & 0 & H_{88}
\end{array}
$$

Figura 2. Matriz hamiltoniana para 3 núcleos

todos os elementos da diagonal são unitários e todos os elementos fora da diagonal são nulos. Em seguida a rotina faz uma série de operações cíclicas iterativas, que podem ser entendidas como rotações, com ambas as matrizes, até que todos os elementos da matriz $A$ que estejam fora da diagonal se tornem nulos. Dizemos que a matriz $A$ foi diagonalizada, e os elementos da diagonal desta matriz $A$ são os autovalores de $H$. As colunas (verticais) da matriz $V$ são os autovetores, ou os coeficientes das funções de onda de cada nível de energia ${ }^{11}$.

Nas Figuras 3 a 5 são mostradas, como ex., uma matriz hamiltoniana numérica ${ }^{12} H$ (Figura 3 ) e sua solução, na forma de matriz $A$ diagonalizada (Figura 4) e matriz $V$ dos autovetores (Figura 5).

Os valores da diagonal de A são os níveis de energia "reais".

\begin{tabular}{c|cccccccc|}
$\mathbf{1}$ & $\mathbf{1}$ & $\mathbf{2}$ & $\mathbf{3}$ & $\mathbf{4}$ & $\mathbf{5}$ & $\mathbf{6}$ & $\mathbf{7}$ & $\mathbf{8}$ \\
$\mathbf{2}$ & $-763,88$ & 0 & 0 & 0 & 0 & 0 & 0 & 0 \\
$\mathbf{3}$ & 0 & $-280,53$ & 6 & 5 & 0 & 0 & 0 & 0 \\
$\mathbf{4}$ & 0 & $-265,48$ & 4 & 0 & 0 & 0 & 0 \\
$\mathbf{5}$ & 0 & 5 & 4 & $-232,88$ & 0 & 0 & 0 & 0 \\
$\mathbf{6}$ & 0 & 0 & 0 & 0 & 229,88 & 4 & 5 & 0 \\
$\mathbf{7}$ & 0 & 0 & 0 & 0 & 5 & 6 & 273,53 & 0 \\
$\mathbf{8}$ & 0 & 0 & 0 & 0 & 0 & 0 & 0 & 778,88
\end{tabular}

Figura 3. Exemplo de matriz hamiltoniana H numérica para 3 núcleos

\begin{tabular}{c|cccccccc|}
$\mathbf{1}$ & $\mathbf{1}$ & $\mathbf{2}$ & $\mathbf{3}$ & $\mathbf{4}$ & $\mathbf{5}$ & $\mathbf{6}$ & $\mathbf{7}$ & $\mathbf{8}$ \\
$\mathbf{2}$ & $-763,88$ & 0 & 0 & 0 & 0 & 0 & 0 & 0 \\
$\mathbf{3}$ & 0 & $-282,87$ & 0 & 0 & 0 & 0 & 0 & 0 \\
$\mathbf{4}$ & 0 & $-264,30$ & 0 & 0 & 0 & 0 & 0 \\
$\mathbf{5}$ & 0 & 0 & 0 & $-231,72$ & 0 & 0 & 0 & 0 \\
$\mathbf{6}$ & 0 & 0 & 0 & 0 & 228,95 & 0 & 0 & 0 \\
$\mathbf{7}$ & 0 & 0 & 0 & 0 & 0 & 0 & 276,67 & 0 \\
$\mathbf{8}$ & 0 & 0 & 0 & 0 & 0 & 0 & 0 & 778,88
\end{tabular}

Figura 4. Exemplo de matriz A numérica diagonalizada (corresponde à matriz H da Figura 3, ou seja, contém os autovalores de H) 


\begin{tabular}{l|cccccccc|}
$\mathbf{1}$ & $\mathbf{1}$ & $\mathbf{2}$ & $\mathbf{3}$ & $\mathbf{4}$ & $\mathbf{5}$ & $\mathbf{6}$ & $\mathbf{7}$ & $\mathbf{8}$ \\
$\mathbf{2}$ & 1,00 & 0 & 0 & 0 & 0 & 0 & 0 & 0 \\
$\mathbf{3}$ & 0 & 0,95 & 0,30 & 0,12 & 0 & 0 & 0 & 0 \\
$\mathbf{4}$ & 0 & $-0,31$ & 0,94 & 0,14 & 0 & 0 & 0 & 0 \\
$\mathbf{5}$ & 0 & $-0,07$ & $-0,17$ & 0,98 & 0 & 0 & 0 & 0 \\
$\mathbf{6}$ & 0 & 0 & 0 & 0,99 & 0,06 & 0,13 & 0 \\
$\mathbf{7}$ & 0 & 0 & 0 & 0 & $-0,11$ & 0,92 & 0,37 & 0 \\
$\mathbf{8}$ & 0 & 0 & 0 & 0 & $-0,10$ & $-0,38$ & 0,92 & 0 \\
& 0 & 0 & 0 & 0 & 0 & 0 & 1,00
\end{tabular}

Figura 5. Exemplo de matriz V numérica (corresponde às matrizes $H$ e A das figuras 3 e 4 , ou seja, contém os autovetores de $H$ )

Para calcular a energia (freqüência) de uma transição entre os níveis $i$ e $j$, basta fazer a diferença

freqüência da transição $=v_{i j}=\left|A_{i i}-A_{j j}\right|$

Para evitar excessivos cálculos inúteis, porém, convém aplicar antes a regra de seleção e depois calcular apenas para os níveis que correspondam a transições permitidas. No caso de 3 núcleos $(8$ níveis), por ex., há 28 transições possíveis ${ }^{13}$, mas apenas 15 são permitidas pela regra de seleção $\Delta m_{T}= \pm 1$. A rotina "Frequencias" calcula $\Delta m_{T}$ para cada possibilidade, usando os valores de $m_{T}$ previamente calculados pela rotina "FinLoopNi"; se o valor absoluto de $\Delta m_{T}$ for 1, ela procede a calcular a frequiência da transição. Para simplificar o procedimento, esta mesma rotina chama também a rotina "VetorTrMat" e a função "Intensidade", que fornecerão as intensidades das bandas; se a intensidade for menor do que o limite estipulado, a banda é desprezada.

Para estes cálculos de intensidades, porém, é necessário que tenha sido construída a matriz de transição antes de iniciar o cálculo das freqüências. O cálculo da matriz de transição é simples, mas envolve uma particularidade interessante: é necessário utilizar uma regra de seleção mais rigorosa, em que se permite mudança de spin para apenas um núcleo. Deve-se observar que a regra de seleção $\Delta m_{T}= \pm 1$ permite que mais de um núcleo tenha sofrido mudança de spin. Na Tabela 1 é possível verificar que as transições entre os níveis $2-7,3-6$ e $4-5$ são permitidas por $\Delta m_{T}= \pm 1$, e envolvem mudanças de três spins simultaneamente. Estas são as chamadas bandas de combinação, que só aparecem em espectros mais complicados, nos quais podem se tornar muito importantes. Nos espectros menos complexos as bandas de combinação não aparecem porque sua intensidade é geralmente pequena. No programa SimEsp_NMR o histograma das bandas de combinação é traçado em cor branca (ou cinza, se for escolhido um fundo branco).

Mas, voltando ao cálculo da matriz de transição, que é efetuado pela rotina "CalcTransMatriz": a rotina compara todos os pares

\begin{tabular}{|c|c|c|c|c|c|c|c|c|}
\hline & 1 & 2 & 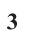 & 4 & 5 & 6 & 7 & 8 \\
\hline 1 & 0 & 1 & 1 & 1 & 0 & 0 & 0 & 0 \\
\hline 2 & 1 & 0 & 0 & 0 & 1 & 1 & 0 & 0 \\
\hline 3 & 1 & 0 & 0 & 0 & 1 & 0 & 1 & 0 \\
\hline 4 & 1 & 0 & 0 & 0 & 0 & 1 & 1 & 0 \\
\hline 5 & 0 & 1 & 1 & 0 & 0 & 0 & 0 & 1 \\
\hline 6 & 0 & 1 & 0 & 1 & 0 & 0 & 0 & 1 \\
\hline 7 & 0 & 0 & 1 & 1 & 0 & 0 & 0 & 1 \\
\hline 8 & 0 & 0 & 0 & 0 & 1 & 1 & 1 & 0 \\
\hline
\end{tabular}

Figura 6. Matriz de transição para o exemplo de três núcleos

possíveis de níveis de energia; ao comparar o nível $k$ com o nível $l$, ela verifica um por um todos os spins $\left(m_{i}(k)\right.$ e $\left.m_{i}(l)\right)$ dos núcleos. Se os dois níveis diferirem apenas pelos spins de um único núcleo, o elemento $\operatorname{TrMat}(k, l)=1$; caso contrário, $\operatorname{TrMat}(k, l)=0$. Evidentemente, esta é uma matriz da mesma ordem das anteriores $\left(2^{p} \times 2^{p}\right)$, e também é simétrica $(\operatorname{TrMat}(k, l)=\operatorname{TrMat}(l, k))$. A Figura 6 mostra a matriz de transição para nosso exemplo. O leitor pode verificar, pela Tabela 1, que ela pode ser construída pelo procedimento descrito acima.

De posse da matriz $V$ e da matriz de transição, a intensidade da transição entre o nível $k$ e o nível $l$ pode ser calculada da seguinte forma: tomamos o vetor (a coluna (vertical) da matriz $V$ ) correspondendo a um dos níveis (4, por ex.), escrevemos este vetor na disposição horizontal e multiplicamos pela matriz de transição. O resultado é um vetor (uma matriz em linha). $\mathrm{O}$ exemplo corresponde aos números da matriz $V$ da Figura 5, e a multiplicação está representada na Figura 7.

$O$ vetor que é o resultado desta multiplicação deve agora ser multiplicado pelo vetor $V$ correspondente ao outro nível (digamos, nível 7): observe-se que devemos tomar o vetor $V$ vertical sempre; para a segunda multiplicação o vetor $V$ é mantido na vertical, como mostrado na Figura 8.

O resultado desta multiplicação, elevado ao quadrado, é a intensidade relativa da transição entre os níveis 4 e $7^{14}$. Naturalmente, se a transição é proibida, o cálculo da intensidade dará zero como resultado; ao invés de aplicar a regra de seleção, poderíamos calcular todas as intensidades e aproveitar apenas aquelas diferentes de zero. Este procedimento, no entanto, envolve um número de operações bem maior, e faria o programa tornar-se lento.

Uma vez calculadas as frequiências e as intensidades de todas as bandas, estas são colocadas em ordem de frequiência decrescente para preencher a tabela (se o usuário tiver optado por não ver a tabela, esta operação não é executada, pois as bandas não precisam estar em ordem para traçar o gráfico).

Em seguida é traçado um histograma, com a particularidade de

$$
\begin{aligned}
& \text { Matriz de Transição }
\end{aligned}
$$

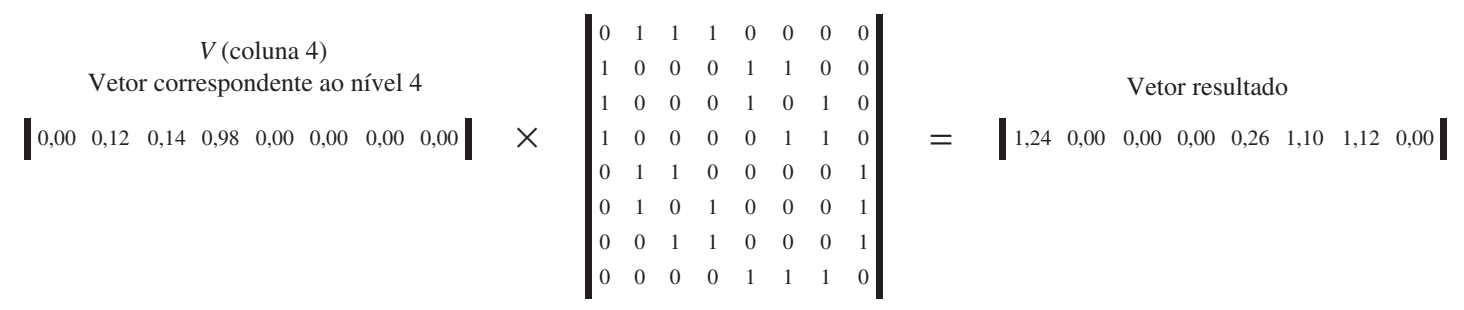




$$
\begin{aligned}
& \text { Vetor } V \text { (coluna 7) }
\end{aligned}
$$

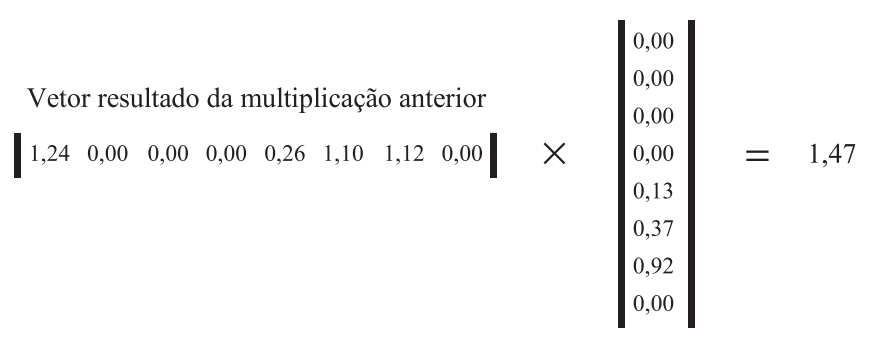

$$
\begin{aligned}
& \text { Intensidade relativa }=(1,47)^{2}=2,16
\end{aligned}
$$

Figura 8. Fim do cálculo de intensidade

que os sinais correspondentes a cada hidrogênio podem ser identificados pela cor. Para alguns espectros simples, o histograma pode já dar uma idéia do aspecto do espectro. Em casos mais complexos (na verdade, na maioria dos casos), porém, é necessário traçar a envolvente calculada de acordo com as leis de distribuição. O programa SimEsp_NMR trabalha tanto com a distribuição de Gauss como de Lorentz, podendo fazer também combinações das duas. Para um único pico de intensidade máxima $I_{\max }$ e freqüência $v_{c}$, as distribuições são calculadas de acordo com as Equações 2 e 3.

$$
\begin{aligned}
& \text { Gaussiana: } y_{g a u}=I_{\max } \times \exp \left[\ln 0,5 \times\left(\frac{\Delta v}{L / 2}\right)^{2}\right] \\
& \text { Lorentziana: } y_{l o r}=I_{\max } \times \frac{1}{1+\left(\frac{\Delta v}{L / 2}\right)^{2}}
\end{aligned}
$$

Nestas equações, $\Delta v=v-v_{c}$ (a distância, em Hz, de cada ponto do gráfico ao centro do pico de absorção, na escala horizontal) e $L$ é a largura (em $\mathrm{Hz})$ do pico na metade de sua altura, um valor que pode ser fornecido pelo usuário, de acordo com a resolução do espectro em questão (o valor "default" é de $1 \mathrm{~Hz}$ ). Quando há vários picos presentes, o valor de $y$ (intensidade) para cada ponto do gráfico é calculado como a soma de todos os valores, correspondentes a todos os picos do espectro. Um número grande de opera- ções é requerido, por isso o número de pontos do espectro é limitado a 963 (o número de pixels, na direção horizontal, da figura apresentada).

Como última operação, o programa examina todos os pontos da envolvente e determina os máximos locais relativos, que são assinalados no gráfico como deslocamentos químicos (em $\mathrm{Hz}$ ).

\section{Utilização do programa}

A utilidade e a eficiência de um programa deste tipo podem, talvez, ser melhor evidenciadas com um exemplo. Em nossas pesquisas sobre síntese de produtos naturais fizemos a preparação do composto 1, mostrado na Figura $9^{15}$.

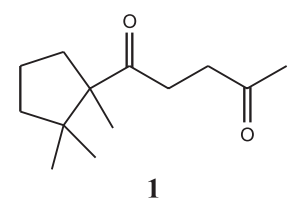

Figura 9. Composto cuja $R M N$ de ${ }^{l} H$ apresenta um sistema $A B C D$

Os quatro hidrogênios dos metilenos que se encontram entre as duas carbonilas apresentam sinais, no espectro de RMN, que correspondem a um sistema $\mathrm{ABCD}$. Esta região do espectro experimental está reproduzida na Figura 10.

Como se vê, apesar de ter sido usado um aparelho de $400 \mathrm{MHz}$, não é possível fazer qualquer interpretação desses dados experimentais fazendo considerações baseadas em espectros de primeira ordem. Com o programa SimEsp_NMR, porém, foi possível fazer a simulação apresentada na Figura 11.

A forte semelhança entre o espectro experimental e o calculado mostra que os dados utilizados no cálculo são bem confiáveis.

Para fazer, porém, uma simulação como esta apresentada na Figura 11 é necessário um trabalho extenso e cuidadoso: o operador precisa ser capaz de retirar do espectro experimental alguns dados que possam servir como pontos de partida apropriados e, depois, tem que fazer inúmeras pequenas modificações até chegar a um resultado satisfatório; é essencial utilizar sinais ( \pm ) corretos

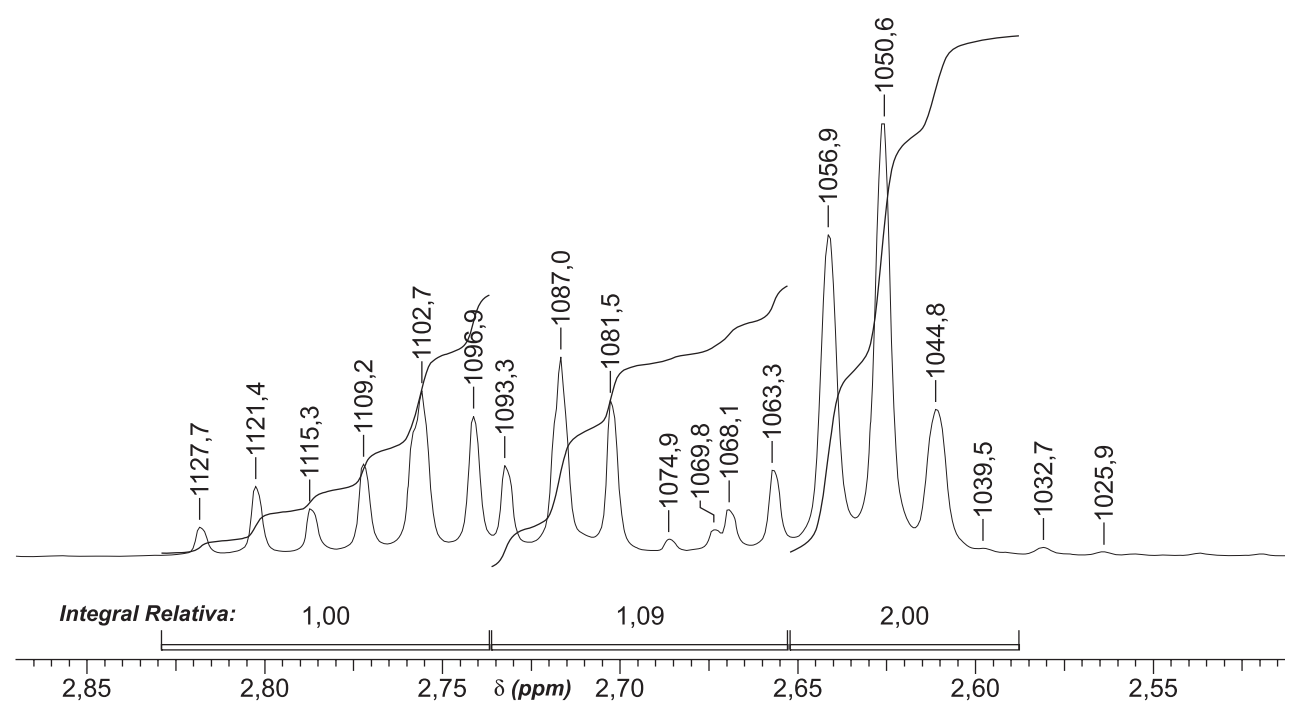

Figura 10. Parte do espectro experimental do composto 1 


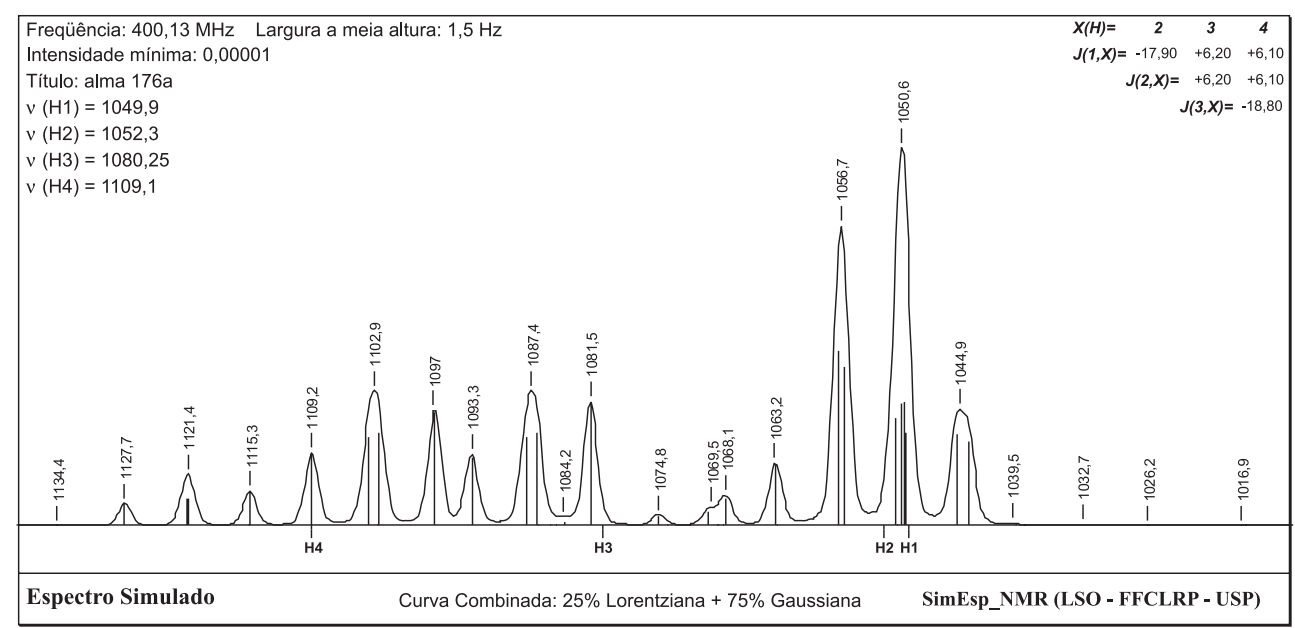

Figura 11. Simulação de um sistema $A B C D$ correspondendo ao do composto 1

para os valores de constantes de acoplamento, e mesmo que dois hidrogênios absorvam na mesma frequiência, o valor da constante de acoplamento entre eles pode ter influência muito importante no resultado final ${ }^{16}$.

Para facilitar essas operações, o programa permite que os dados usados em uma simulação (deslocamentos químicos, valores de J, etc.) sejam gravados em arquivos do tipo texto (com extensão particular do programa, *.esm); durante esses trabalhos de ajuste, o usuário pode ir salvando quantas versões desejar, sem correr o risco de perder "aquela simulação que estava quase boa", porque o programa pode ler os arquivos e recuperar os dados para reiniciar as modificações.

Um aspecto também importante é o seguinte: já foi mencionado que o número de pontos do gráfico é limitado a 963; este é o total de pontos para todo o espectro. Em um caso como o da Figura 11, em que todos os hidrogênios estão contidos em uma faixa estreita de freqüências, não há problema: o número de pontos é perfeitamente satisfatório. Em um caso, porém, em que dois hidrogênios em 3,5 e 3,6 $\delta$ estivessem acoplados com um $\mathrm{CH}_{3}$ em $0,8 \delta$, o espectro é traçado em toda a faixa, ficando com poucos pontos em uma expansão da região 3,5-3,6 $\delta$. Para solucionar este problema, é possível expandir e centralizar qualquer região de interesse e clicar no botão "Fixar Janela"; o programa passará a trabalhar com essa janela fixa, e esta região conterá agora todos os pontos do espectro, melhorando muito a aparência dos picos e a exatidão dos deslocamentos químicos correspondentes aos máximos da envolvente.

Vários outros aspectos poderiam ser destacados, mas acreditamos que os leitores interessados poderão aproveitar muito melhor as informações que retirarem do uso e da experimentação com o próprio programa. O programa é inofensivo, não contém rotinas que possam causar dano ao computador ou a outros programas: sugerimos aos interessados que experimentem, clicando em todos os botões e abrindo todos os menus, para descobrir tudo o que o programa pode fazer.

Esperamos especialmente que a disponibilidade dessas rotinas anime os químicos com inclinação para programação a desenvolverem programas que sejam adequados a suas necessidades particulares.

\section{AGRADECIMENTOS}

À FAPESP, à CAPES e ao CNPq pelo apoio financeiro.

\section{REFERÊNCIAS E NOTAS}

1. Este programa, também desenvolvido no Laboratório de Síntese Orgânica da FFCLRP/USP, pode ser baixado sem restrições da mesma página http:// artemis.ffclrp.usp.br/NMR.htm (o programa chama-se FOMSC3.zip, e tem 2,53 MB).

2. As rotinas que produzem o gráfico (a principal delas chama-se "Grafico") podem surpreender porque contêm grande número de funções da API do Windows: isto é necessário porque a mesma rotina é usada tanto para fazer o gráfico na tela como para gerar o arquivo metafile, que produzirá a cópia do espectro de boa qualidade gráfica. Para gerar o arquivo metafile é essencial utilizar as funções da API do Windows.

3. Outros arquivos (alternativos) que podem ser copiados são os seguintes: SimEsp_NMR_(exe).zip (80,7 KB); SimEsp_NMR_texto(doc).zip $(65,0 \mathrm{~KB})$ e SimEsp_NMR_texto(txt).zip $(27,5 \mathrm{~KB})$

O primeiro destes contém apenas o arquivo executável; é o programa completo e pode rodar em muitos computadores, sem requerer instalação, tendo a vantagem de ser bem pequeno. No entanto, se o computador não contiver certos arquivos, o executável não poderá rodar, e é então necessário baixar o programa compilado (SimEsp_NMR_Compil.zip) para instalar. Algumas versões do Windows apresentam um problema com alguns arquivos do sistema (quando os arquivos existem mas são de versões diferentes) que impede a instalação deste programa (o Windows não permite que o programa de instalação substitua o arquivo antigo). Estamos procurando uma solução mais geral para o problema, mas uma solução provisória satisfatória consiste em usar os pacotes alternativos SimEsp_NMR_Compil_SAS.zip ou SimEsp_NMR_W98. Ambos contêm o mesmo programa, mas o primeiro não tenta instalar os arquivos do sistema que praticamente todos os computadores já têm, e o segundo contém arquivos mais antigos, tendo menor probabilidade de gerar problemas.

4. Há uma certa confusão na literatura sobre a atribuição das denominações $\alpha$ e $\beta$ às funções de onda com $m=+1 / 2$ ou $-1 / 2$. Estamos utilizando aqui a representação recomendada em Parameters and Symbols for Use in Nuclear Magnetic Resonance (IUPAC Recommendations 1997), em Harris, R. K.; Kowalewski, J.; Menezes, S. C.; Pure Appl. Chem. 1997, 69, 2489.

5. O fato de $m=+1 / 2$ corresponder ao nível de energia mais baixo é também conseqüência das definiçõoes da IUPAC mencionadas na nota 4, pois $m=$ $+1 / 2$ corresponde à orientação no mesmo sentido do campo magnético aplicado. Uma maneira mais simples de interpretar isto vem do fato de que a energia de magnetização é $E=-m \gamma \hbar B_{0}$; o sinal negativo deixa evidente que $m=+1 / 2$ corresponde ao nível mais baixo (quando $\gamma>0$, como é o caso para o hidrogênio).

6. Para tornar o texto mais claro, vários aspectos foram aqui deixados de lado. A simplificação utilizada justifica-se essencialmente pelo fato de que estamos presumindo que a frequiência de absorção $v_{i}$ de um hidrogênio é conhecida (por ex., por ter sido determinada experimentalmente), e estamos interessados apenas nas diferenças de energia entre os vários níveis. Se um núcleo de energia $E_{\mathrm{o}}$ for colocado em um campo magnético, poderá existir em dois estados, de energias $E_{0}+1 / 2 E_{\text {mag }}$ e $E_{0}-1 / 2 E_{\text {mag }}$; a diferença de energia entre esses dois estados é $E_{\text {mag }}$, e é equivalente a $h v_{i}$ (a energia do fóton que promove a transição). Podemos dizer que a energia de cada 
estado é $E_{\mathrm{o}}+1 / 2 h v_{i}$ e $E_{\mathrm{o}}-1 / 2 h v_{i}$; suprimindo o valor constante $E_{\mathrm{o}}$, porque só nos interessam as diferenças, chegamos às expressões apresentadas no texto (exceto pela questão dos sinais, discutida logo adiante). Sem grande esforço, é possível estender o raciocínio para o caso de vários núcleos onde, nos vários níveis, os termos constantes apareceriam somados.

É evidente que, nas expressões acima, os valores $+1 / 2 e-1 / 2$ resultam dos valores de $m$. No entanto, devido às convenções adotadas, a energia envolvida no processo é dada por $E=-m \gamma \hbar B_{0}$, de forma que temos que fazer a equivalência acrescentando um sinal negativo, $E=-m h v$, caso contrário os valores de $m$ pareceriam estar invertidos.

7. O procedimento descrito corresponde a uma aplicação do método variacional para a solução do sistema em questão. Como exemplos de referências, pode-se consultar: Levitt, M. H.; Spin Dynamics, John Wiley \& Sons: New York, 2001; Günther, H.; NMR Spectroscopy, An Introduction, John Wiley \& Sons: New York, 1987; Friebolin, H.; Basic One- and TwoDimensional NMR Spectroscopy, VCH: Weinheim, 1991; Gil, V. M. S.; Geraldes, C. F. G. C.; Ressonância Magnética Nuclear, Fundação Calouste Gulbenkian: Lisboa, 1987; Bothner-By, A. A.; Castellano, S. M. Em Computer Programs for Chemistry; Detar, D. F., ed.; W. A. Benjamin: New York, 1968, vol. 1; Garbisch, Jr., E. W.; J. Chem. Educ. 1968, 45, 311; Garbisch, Jr., E. W.; J. Chem. Educ. 1968, 45, 402; Garbisch, Jr., E. W.; J. Chem. Educ. 1968, 45, 480. Há um grande número de publicações sobre a matéria em periódicos de Química, e também muita informação disponível na Internet.

8. Naturalmente, neste processo a rotina também transfere adequadamente os valores de $m_{i}$ e de $m_{T}$

9. Em textos teóricos, diz-se que os elementos $H_{k l}$ são calculados pelo operador $H_{k l}=U_{k l} J_{i,}$, onde $U_{k l}$ tem o valor $+1 / 2$ se as funções de onda $\Psi_{k} \mathrm{e}$ $\Psi$, diferirem exclusivamente pela troca das coordenadas dos spins $i$ e $j$; em todos os outros casos, $U_{k l}=0$. Os "critérios" mencionados no texto traduzem isto para termos práticos.

10. Em nosso programa utilizamos a rotina JACOBI, escrita por Ed Wilson em dezembro de 1990 e publicada em http://www.csiberkeley.com/ Tech_Info/d.pdf. A rotina foi traduzida de FORTRAN para BASIC por I. A. Borin. Fizemos apenas uma modificação muito pequena para evitar, em certos casos, uma divisão por zero que resultava em erro e interrupção do programa.

11. As matrizes têm sempre valores tais que $H V=V A$. Além disso, para cada coluna da matriz $V$, a soma dos quadrados dos elementos é igual à unidade,

$\sum c_{i}^{2}=1$. Outras verificações úteis são: o determinante da matriz $H$ é igual a o produto dos autovalores (elementos da diagonal de $A$ ); a soma dos elementos da diagonal (traço das matrizes) é igual a zero, tanto para a matriz $H$ como para a matriz $A$.

Deve-se observar que a matriz hamiltoniana pode ser submetida diretamente à rotina JACOBI mas, para reduzir o volume de cálculos, o programa divide a matriz $H$ em sub-matrizes, que são submetidas separadamente; isto é possível porque grande parte dos elementos dessas matrizes são elementos nulos, que não modificam o resultado mas aumentam o número de operações. O leitor interessado pode ver as sub-matrizes ao rodar o programa SimEsp_NMR, usando o menu "Janela", sub-menu "Matrizes". A variedade de cores usadas na exibição das matrizes permite visualizar facilmente as sub-matrizes.

12. Para esta matriz foi utilizada uma frequiência não realista, para um aparelho de $\mathrm{RMN}$ de ${ }^{1} \mathrm{H}$, de $0,0003 \mathrm{MHz}$, apenas para manter os números da diagonal em tamanho conveniente para impressão. O leitor pode observar resultados reais utilizando o programa SimEsp_NMR (como na nota anterior, menu "Janela", sub-menu "Matrizes").

13. $C_{8}^{2}$, número de combinações de 8 elementos tomados 2 a 2 . O número pode ser calculado por qualquer das fórmulas: $C_{n}^{p}=\frac{n(n-1) \ldots(n-p+1)}{1.2 .3 \ldots p}$ ou $C_{n}^{p}=\frac{n !}{p !(n-p) !}$

14. Esta seqüência de operações para cálculo das intensidades consiste na tradução prática da expressão $P_{k l}=\left(\sum_{i} \sum_{j} c_{i k} T_{i j} c_{j l}\right)^{2}$, onde $c_{i k}$ e $c_{j l}$ são os coeficientes das funções de onda $\Psi_{k} \mathrm{e} \Psi_{l}$ (os valores da coluna $k$ e da coluna $l$ na matriz $V$ ), e $T_{i j}$ tem valor 1 se $\Psi_{i}$ e $\Psi_{j}$ diferirem pelo spin de apenas um núcleo, tendo valor 0 em qualquer outro caso.

15. Araujo, A. L. S. M.; Tese de Doutoramento, Faculdade de Filosofia, Ciências e Letras de Ribeirão Preto, Universidade de São Paulo, Brasil, 2004 ou em http://artemis.ffclrp.usp.br/TesesPDF/AnaDoutCorr.pdf; na tese este composto é identificado pelo número 47.

16. A análise de um espectro de RMN de ${ }^{1} \mathrm{H}$ é uma arte complexa e delicada; cada espectro tem suas próprias características e precisa ser analisado individualmente. Um bom ponto de partida é o exame cuidadoso da integral. Neste nosso caso a integral mostra que os sinais dos quatro hidrogênios podem estar divididos em três grupos: 1 hidrogênio, 1 hidrogênio e 2 hidrogênios (possivelmente os dois que estão juntos pertencendo ao mesmo metileno). Podemos, então, iniciar (de forma um tanto simplificada) a nossa simulação por colocar cada hidrogênio no centro dos sinais que correspondem a eles. Os valores de constantes de acoplamento iniciais podem também ser valores médios correspondentes à estrutura esperada ou imaginada, valores que podem ser retirados de tabelas; neste nosso caso devemos ter $J_{\text {gem }}(-12 \mathrm{a}-20 \mathrm{~Hz})$ e $J_{\text {vic }}(\approx+7 \mathrm{~Hz})$ para compostos acíclicos. Depois comparamos o espectro simulado com o calculado. Se todos os picos de um hidrogênio estão deslocados para o mesmo lado, o deslocamento químico do hidrogênio deve estar muito alto ou muito baixo, e precisa ser corrigido. Se há picos (do mesmo hidrogênio) mais afastados um do outro do que no espectro experimental, é preciso reduzir algum valor de $J$. Há muitos outros aspectos que podem ser considerados mas que não cabem aqui. Uma análise mais detalhada deste mesmo espectro pode ser encontrada em http://artemis.ffclrp.usp.br/ NMR.htm com o nome de "Interpretação.pdf". 